\title{
Preparation and evaluation of polymeric beads composed of Chitosan-Gellan Gum-Gum Ghatti/-Gum Karaya polyelectrolyte complexes as polymeric carrier for enteric sustained delivery of Diclofenac sodium
}

\author{
Anurag Verma ${ }^{1 *}$, Pradeep Kumar $^{2}$, Vaibhav Rastogi ${ }^{2}$ and Piyush Mittal ${ }^{1}$
}

\begin{abstract}
Background: An enteric coating is a multistep technique that involves deposition of a polymeric barrier over uncoated orally administered tablets to prevent them from dissolving or disintegrating in the stomach. However, as soon as the coating dissolves in the alkaline environment of intestine, the whole of the drug come in direct contact with gastric mucosa leading to irritation to distal parts of the gastrointestinal tract (GIT). Considering the above facts, there is clear need to develop a simple and effective enteric release formulation for gastric irritant drugs like Diclofenac sodium (DS). The goal of this study was to create enteric release polymeric polyelectrolyte complex (PEC) beads made up of cationic Chitosan (CH) and anionic Gellan Gum (GG) for sustained DS delivery to the intestine. The beads were prepared by extruding a solution of GG and Gum Ghatti (GT) or GG and Gum Karaya (GK) bearing DS into $\mathrm{CH}$ solution in $1 \% \mathrm{w} / \mathrm{v}$ acetic acid, with the help of a syringe fitted with a 18 gauge hypodermic needle.

Results: Instantly created spherical beads were dried in a hot air oven $60^{\circ} \mathrm{C}$ overnight. In $0.1 \mathrm{M} \mathrm{HCl}$ and $6.8 \mathrm{pH}$ phosphate buffer, the dried beads were tested for drug entrapment in the beads, in vitro swelling of beads and in vitro drug release studies from the beads. The \% drug entrapment efficiencies (\% DEE) of these PEC beads ranged from $59.54 \pm 2.09$ to $81.03 \pm 4.22 \%$. In $0.1 \mathrm{M} \mathrm{HCl}$, the PEC beads swelled the least in vitro, but expanded significantly in phosphate buffer ( $\mathrm{pH}$ 6.8). The in vitro release of Diclofenac sodium from different PEC beads in $0.1 \mathrm{M} \mathrm{HCl}$ was found to be less than 7.5 percent, whereas the release was sustained for $6 \mathrm{~h}$ in phosphate buffer ( $\mathrm{pH} 6.8$ ).
\end{abstract}

Conclusions: From the experimental data, it may be concluded that these PEC beads can be useful as potential multiple-unit enteric release polymeric carrier systems for sustained delivery of gastric irritant drugs like Diclofenac sodium.

Keywords: Chitosan, Gum Ghatti, Gum Karaya, Gellan Gum, Polyelectrolyte complex, Drug release

${ }^{*}$ Correspondence: anuragverma_iftm@yahoo.co.in

${ }^{1}$ Teerthanker Mahaveer College of Pharmacy, Teerthanker Mahaveer

University, Moradabad, Uttar Pradesh 244001, India

Full list of author information is available at the end of the article

\begin{abstract}
Background
Nonsteroidal anti-inflammatory drugs (NSAIDs) have long been recognized as having significant therapeutic efficacy, particularly in the treatment of inflammatory illnesses such as osteoarthritis (OA) and rheumatoid arthritis (RA). Diclofenac sodium (DS) is a type of NSAID that is exceptionally strong and effective as an analgesic
\end{abstract}


and anti-inflammatory medication. It's one of the most often given nonsteroidal anti-inflammatory medications on the planet (1). The symptomatic treatment of rheumatoid arthritis, osteoarthritis, and ankylosing spondylitis is indicated with DS. It's also used to treat acute musculoskeletal injuries, acute shoulder pain, postoperative pain, and dysmenorrhea in the short term. However, DS causes negative effects in roughly $20 \%$ of individuals, necessitating prescription discontinuation. Gastrointestinal hemorrhage and abnormally high liver enzymes are among the side effects $[1,2]$.

Because of its short biological half-life (less than $2 \mathrm{~h}$ ) and rapid clearance rate (mean elimination half-life $1.2-1.8 \mathrm{~h}$ ), DS must be given often to maintain therapeutic concentrations, which may raise the risk of adverse effects. Modified-release dosage forms are essential to reduce stomach damage and make the Diclofenac dosing regimen safer and more convenient for patients. [3, 4]. Most commonly used oral DS formulations available in the market are enteric-coated tablets. Enteric coating is a specialized technique which requires sophisticated instrumentation and thus very expensive. Another problem associated with enteric coated products is the fact that as soon as the coating dissolves, the whole of the drug come in direct contact with gastric mucosa leading to irritation to distal parts of the gastrointestinal tract (GIT) [5]. Given the foregoing, it is clear that a simple and effective enteric release formulation for DS is required, in which the drug is released in a $\mathrm{pH} 6.8$ mixed phosphate buffer from an enteric polymer matrix in a sustained manner, with the negligible release in the stomach's acidic $\mathrm{pH}(0.1 \mathrm{M} \mathrm{HCl}, \mathrm{pH} 1.2)$, reducing the risk of gastric irritation. In the quest to find out suitable polymer matrix, we have carried out some preparatory work in our laboratory exploring the potential of Gellan gumChitosan (GG-CH) polyelectrolyte complexes (PECs) beads as an enteric polymer matrix. The fact that PECs generated between a polycation and a polyanion have a high degree of ordering and crystal-like qualities, as well as compact structures, is why they were chosen as a polymeric carrier. Because these PEC beads will be composed of nontoxic biopolymers, they are expected to have a variety of unique physicochemical features of various polyelectrolytes [6, 7]. Their formation may occur through one or more of the following mechanism: the coulombic attraction or other interactions such as hydrogen bonding, charge transfer interaction, dipole-dipole interaction, van der Waal's interaction and hydrophobic effect $[8,9]$. The formations of PECs avoid the use of chemical cross-linkers. Therefore, it is expected that these PECs are devoid of any possible toxicity and other undesirable effects of chemical cross-linkers [9]. PEC beads were prepared by dropping the GG ( $1 \% \mathrm{w} / \mathrm{v}$ in deionized water) into the acidic Chitosan solution $(0.4 \% \mathrm{w} / \mathrm{v}$ in $1 \% \mathrm{w} / \mathrm{v}$ acetic acid). The beads were formed instantaneously. We have observed that dried GG-CH PEC beads swell very little in acidic $\mathrm{pH}$, but swelled to a great extent in the intestinal environment ( $\mathrm{pH} 6.8$, mixed phosphate buffer). However, these beads were not able to curtail the release of DS in acidic dissolution medium with more than $10 \%$ DS was released in $120 \mathrm{~min}$.

After this observation, in order to increase the strength of GG-CH PEC beads we have decided to combine the GG with anionic Gum Ghatti or Gum Karaya in varying concentration, keeping the GG concentration constant. Recently, numerous PECs made of various ionic polysaccharides have been extensively investigated for the use in various biomedical applications such as drug delivery, tissue engineering, enzyme immobilization, and biosensor. $[6,10]$. Considering the versatility of PECs, in the present study, an attempt was made to explore the utility of PECs comprised of a cationic polymer, Chitosan and anionic polymers such as Gum Ghatti and Gum Karaya in combination with Gellan Gum as release retarding enteric polymeric carrier for the oral enteric delivery of Diclofenac sodium.

\section{Methods}

\section{Materials}

Diclofenac sodium (DS) was obtained as gift sample from Unique Life Sciences, India. Deacetylated Gellan Gum (Gelzan ${ }^{\mathrm{TM}} \mathrm{CM}, \mathrm{DD}=$ ) was purchased from SigmaAldrich. Chitosan (Viscosity 25 cps; $0.4 \%$ solution in $1 \%$ acetic acid at $26^{\circ} \mathrm{C}$; degree of deacetylation $=\geq 75 \%$ ) was obtained as gift sample from Central Institute of Fisheries Technology, Cochin, India. Gum Ghatti and Gum Karaya were purchased from Loba Chemie Pvt. Ltd., India. Double-distilled water was used throughout the study. All other reagents were of analytical grade.

\section{Preparation of Gellan gum-Gum Ghatti and Gellan gum- Gum Karaya solutions}

The solutions were made by dispersing the appropriate gum mixtures in deionized water and then heating the system to $90{ }^{\circ} \mathrm{C}$ with constant stirring until it became homogeneous.

\section{Preparation of drug polymer mixture}

After the preparation of homogenous aqueous polymeric dispersion, DS was added into it. It was observed that addition of DS into the almost semisolid polymeric dispersion, the dispersion become fluid at system room temperature $\left(28^{\circ} \mathrm{C}\right)$. 


\section{Preparation of Chitosan solution}

The needed amount of Chitosan ( 0.4 percent w/v) was added to a 1 percent acetic acid solution in deionized water, and the combination was maintained at room temperature overnight for complete polymer ionization.

\section{Preparation of PEC beads}

A simple ionotropic gelation process was used to make the polymeric beads. Using a hypodermic syringe fitted with an $18 \mathrm{G}$ needle, mixtures of homogeneous dispersions $(10 \mathrm{ml})$ of anionic polymer-blends (Gellan GumGum Ghatti or Gellan Gum-Gum Karaya) with or without drug were extruded into the gelation medium composed of Chitosan (0.4 percent $\mathrm{w} / \mathrm{v}$ in 1 percent $\mathrm{w} / \mathrm{v}$ acetic acid, volume $100 \mathrm{ml}$ (Table 1 ). The produced beads were then allowed to cure in the Chitosan solution for $30 \mathrm{~min}$ before being strained and thoroughly cleaned with deionized water until they were free of surface adherent drug. compression machine. The sample pellets were placed in an FTIR compartment, and spectrum scanning was performed using an FTIR at wavelengths ranging from 450 to $4000 \mathrm{~cm}^{-1}$.

\section{\%Drug entrapment efficiency (\%DEE) One hundred $\mathrm{mg}$ of Diclofenac sodium-containing PEC beads was taken and smashed with pestle and mortar}

These beads were crushed and placed in flasks with $50 \mathrm{cc}$ of phosphate buffer ( $\mathrm{pH}$ 6.8). The flasks were shaken for $2 \mathrm{~h}$ with an orbital shaker to extract the DS entirely. After that, the solutions were filtered using Whatman ${ }^{\circledR}$ filter paper (No. 40) and the filtrate was centrifuged to remove the polymeric debris using a tabletop centrifuge (Remi Motors, India). The polymeric debris was then rinsed twice with fresh solvent (water) to remove any DS that had clung to it. A UV-VIS spectrophotometer (Shimadzu 1800, Japan) was used to measure the DS content of the clear supernatant solution at a wavelength of $285 \mathrm{~nm}$. The drug entrapment efficiencies (\%) of various PEC beads containing Diclofenac sodium were calculated using this formula:

$\% \mathrm{DEE}=($ experimental drug loading $/$ Theoretical drug loading $) \times 100$

\section{Confirmation of PEC formation and drug-excipient interaction studies}

The PEC generation and drug-excipient interactions were studied using Fourier transform spectroscopy (FTIR) and Differential Scanning Calorimetry. To eliminate the moisture, the samples were heated. In the DSC analysis, samples $(5 \mathrm{mg})$ were placed in hermetically sealed crimped aluminium pans. Thermograms were taken at a rate of $5{ }^{\circ} \mathrm{C}$ per minute. A Differential Scanning Calorimeter was used to conduct these tests (Universal V4.5A TA Instrument). $\mathrm{KBr}$ pellets of approximately $1 \mathrm{~mm}$ diameter were produced for FTIR characterization by grinding $3-5 \mathrm{mg}$ of samples with $100-150 \mathrm{mg}$ of $\mathrm{KBr}$ in a pressure

\section{In vitro swelling studies}

The swelling behavior of PEC beads was studied using $0.1 \mathrm{M} \mathrm{HCl}$ and phosphate buffer at $\mathrm{pH} 1.2$ and 6.8 , respectively. The pre-weighed amount (100 mg) of PEC beads was immersed in $0.1 \mathrm{M} \mathrm{HCl}(\mathrm{pH} \mathrm{1.2)}$ for $2 \mathrm{~h}$ and in phosphate buffer (pH 6.8) for $6 \mathrm{~h}$ in vessels of dissolution apparatus USP paddle type (Electrolab TDP-08L, India) containing $900 \mathrm{ml}$ respective medium. The swelling experiment was carried out at $37{ }^{\circ} \mathrm{C}$ with a paddle speed of $50 \mathrm{rpm}$. The swelling beads were removed on a regular basis and weighed after tissue paper was used to dry the surface. The weight change of these PEC beads was used to determine the swelling behavior of the beads. The following formula was used to calculate the swelling index $[7,8]$ :

$$
\text { Swelling index }(\%)=\frac{\text { Weight of beads after swelling }- \text { Dry weight of beads }}{\text { Dry weight of beads }} \times 100
$$

Table 1 Formulation composition of different chitosan-based PEC beads containing Diclofenac sodium along with drug entrapment efficiency and swelling results

\begin{tabular}{llllll}
\hline Formulation code & Gellan gum* & Gum Ghatti* & Gum Karaya* & DS* $^{*}$ & Chitosan* $^{*}$ \\
\hline P2 & 1.0 & 1.0 & - & 2.0 & 0.4 \\
P3 & 1.0 & 1.5 & - & 2.0 & 0.4 \\
P4 & 1.0 & 2.0 & - & 2.0 & 0.4 \\
P5 & 1.0 & - & 1.0 & 2.0 & 0.4 \\
P6 & 1.0 & - & 1.5 & 2.0 & 0.4 \\
\hline
\end{tabular}




\section{In vitro drug release studies}

The in vitro release of DS from PEC beads was carried out for $6 \mathrm{~h}$ in a dissolution apparatus of the USP paddle type (Electrolab TDP-08L, India) containing $900 \mathrm{ml}$ of phosphate buffer ( $\mathrm{pH}$ 6.8) as dissolution medium, kept at $370.5^{\circ} \mathrm{C}$ with a stirring speed of $50 \mathrm{rpm}$ and maintained at $370.5{ }^{\circ} \mathrm{C}$. Each time, $5 \mathrm{ml}$ of aliquot was withdrawn at regular intervals. Every sample time, the volume of the dissolving medium was adjusted to $900 \mathrm{ml}$ by replacing $5 \mathrm{ml}$ of phosphate buffer ( $\mathrm{pH}$ 6.8). The absorbance was measured using a UV-VIS spectrophotometer (Shimadzu 1800, Japan) at $285 \mathrm{~nm}$ after the aliquots were filtered and diluted appropriately.

\section{Drug release kinetics}

Drug release kinetics can be interpreted using a variety of kinetic models, including zero-order, first-order, and square root (Higuchi). A zero-order release refers to the release of a drug from a solid dosage form into an aqueous environment at a constant or nearly constant rate, regardless of the drug concentration in the dosage form at any given time.

Dosage formulations with zero-order release provide the most therapeutic benefit with the fewest negative effects. Many extended release formulations have a quick rise in drug release followed by a rapid drop in drug release. The first-order release is the most common sort of medication release. Such a dosage form may not maintain consistent medication concentration levels in the system. $[9,10]$. According to the Higuchi release equation, drug release is mostly driven by diffusion:

$$
Q=K \sqrt{t},
$$

where $Q$ is the amount of medication released in time $t$ and $K$ is the equation's release constant. As shown in equation, the data were additionally treated to the Korsmeyer-Peppas power law (2). The Korsmeyer-Peppas model elucidates the type of drug release mechanism that occurs when swellable polymeric matrices are used. [11, 12]:

$$
\frac{M t}{M \infty}=K t^{n},
$$

time $t, K$ is the structural and geometric constant, and $n$, the release exponent, is calculated from the logarithmic release data using linear regression. In practice, regardless of the physical shape of the delivery device, the first $60 \%$ of a release curve must be used to estimate the slope obtained from equation [2]. The combined effect of diffusion and relaxation mechanisms for the release is shown by a good fit to the Korsmeyer-Peppas equation.

\section{Statistical analysis}

All data were analyzed and expressed as mean, standard deviation using MedCalc software, version 11.6.1.0. (S.D.). Each measurement was carried out three times $(n=3)$.

\section{Results \\ Confirmation of Chitosan-Gellan Gum-Gum Ghatti and -Gum Karaya PEC beads formation DSC analysis}

Figure 1 shows the DSC thermograms of Chitosan, Gellan Gum, Gum Ghatti, and Gum Karaya, as well as dried blank Chitosan-Gellan Gum-Gum Ghatti beads and dried blank Chitosan-Gellan Gum-Gum Karaya beads. Chitosan's DSC thermogram revealed a large endothermic peak at around $71.01{ }^{\circ} \mathrm{C}$, which corresponded to water vaporization (Fig. 1a). It also showed a large exothermic peak at $298.03{ }^{\circ} \mathrm{C}$, which corresponded to sluggish Chitosan breakdown. A wide endothermic peak was observed in the DSC thermogram of pure Gellan Gum at $75^{\circ} \mathrm{C}$, which corresponded to the loss of water (Fig. 1b). There was also an exothermic peak at roughly $245{ }^{\circ} \mathrm{C}$, indicating that the Gellan Gum was degrading. At $192.81{ }^{\circ} \mathrm{C}, 209.81{ }^{\circ} \mathrm{C}$, and $332.25^{\circ} \mathrm{C}$, the DSC thermogram of pure Gum Ghatti revealed three endothermic peaks (Fig. 1c). The first endothermic peak can be attributed to water loss owing to vaporization, but the second and the third endothermic peak can be attributed to twostage decomposition, with the beginning and ultimate decomposition of the polymer occurring at $209.81{ }^{\circ} \mathrm{C}$ and $332.25{ }^{\circ} \mathrm{C}$, respectively. The DSC thermogram of Gum Karaya revealed a sharp endothermic peak at $52.88{ }^{\circ} \mathrm{C}$, which corresponded to water loss, and a broad endothermic peak at $316{ }^{\circ} \mathrm{C}$, which corresponded to Gum Karaya degradation (Fig. 1d). The DSC thermogram of dried blank Chitosan-Gellan Gum-Gum Ghatti beads revealed an endothermic peak at $230{ }^{\circ} \mathrm{C}$, which corresponded to polymeric-matrix degradation of ChitosanGellan Gum-Gum Ghatti beads. Ghatti (Fig. 1e).

The DSC thermogram of dried blank Chitosan-Gellan Gum-Gum Karaya beads revealed a large endothermic peak at $219.29{ }^{\circ} \mathrm{C}$, which corresponded to polymericmatrix degradation of Chitosan-Gellan Gum-Gum Karaya (Fig. 1f).

\section{Fourier transform-infrared (FTIR) spectroscopy analyses}

Figure 2 depicts the FTIR spectra of Chitosan, Gum Ghatti, Gum Karaya, dried blank Chitosan-Gellan Gum-Gum Ghatti beads, and dried blank Chitosan-Gellan GumGum Ghatti beads. Chitosan's FTIR spectra revealed absorption bands at 1650.85, 1379.50, and $1315.32 \mathrm{~cm}^{-1}$, respectively, attributed to amide I, II, and III (Fig. 2a). The hydroxyl stretch and $\mathrm{C}-\mathrm{H}$ stretch caused absorption bands 

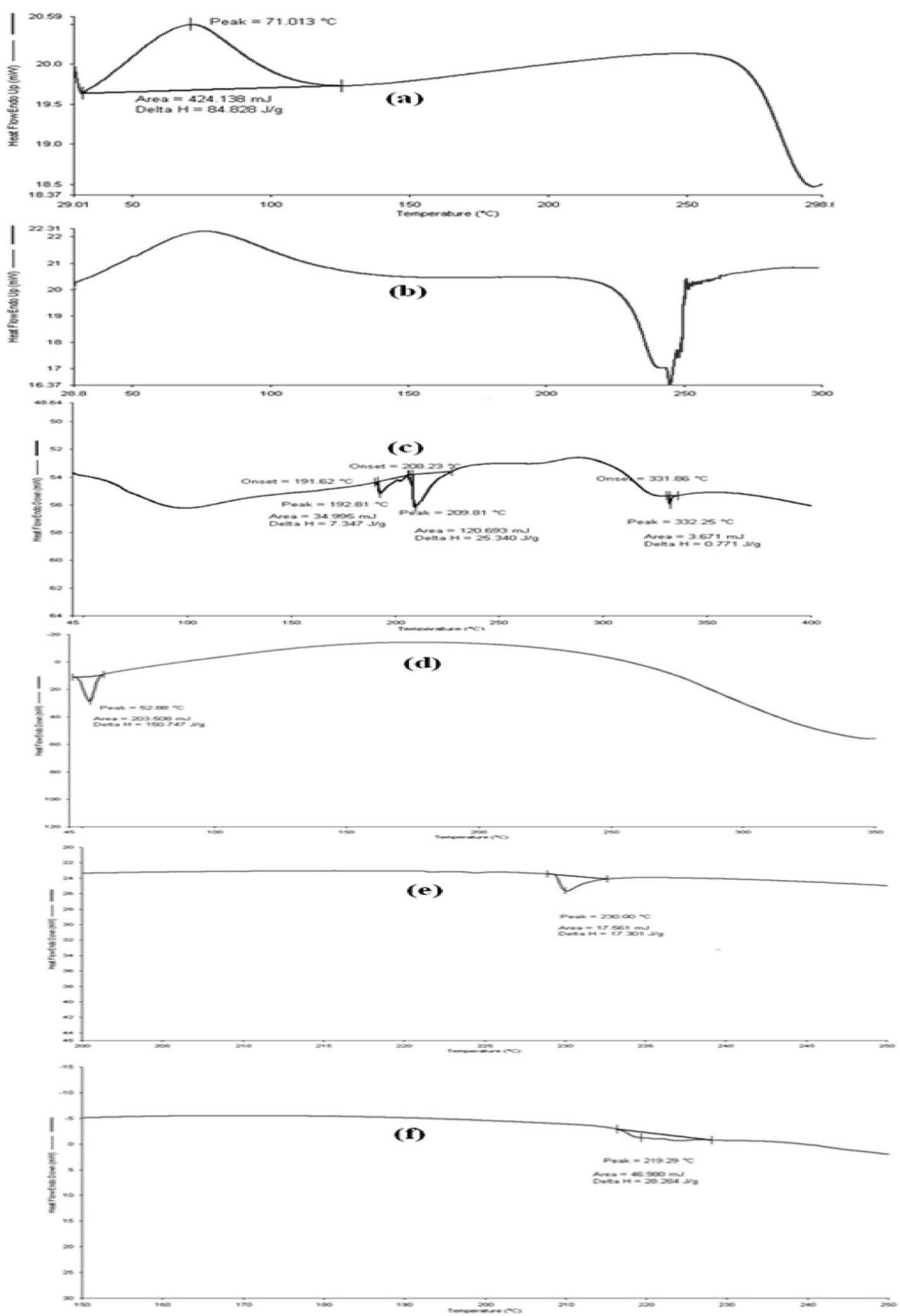

Fig. 1 DSC thermograms of Chitosan (a), Gellan gum (b), Gum Ghatti (c), Gum Karaya (d), dried blank Chitosan-Gellan Gum-Gum Ghatti PEC beads (e) and dried blank Chitosan-Gellan Gum-Gum Ghatti PEC beads (f) 

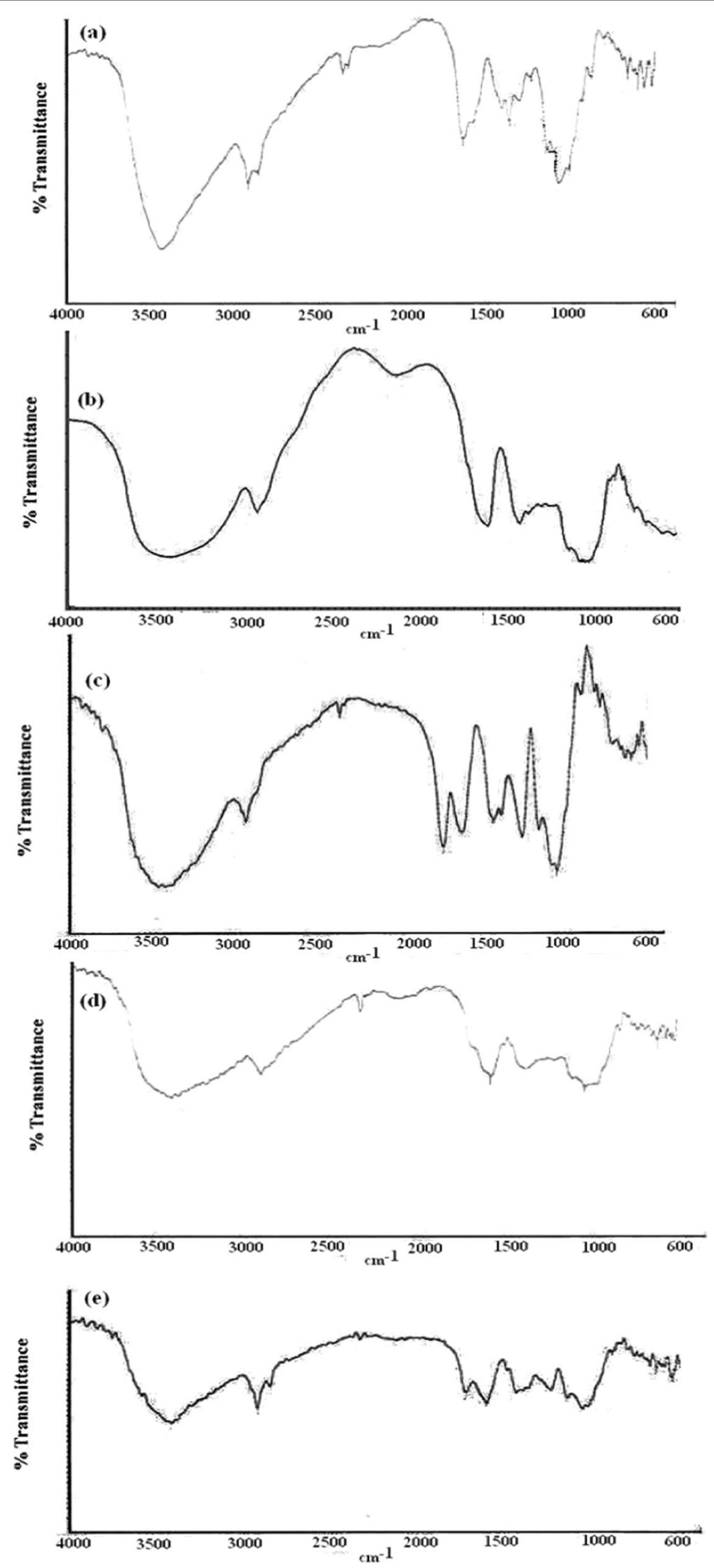

Fig. 2 FTIR spectra of Chitosan (a), Gum Ghatti (b), Gum Karaya (c), dried blank Chitosan-Gellan Gum-Gum Ghatti PEC beads (d) and dried blank chitosan-Gellan gum-gum Karaya PEC beads (e) 
at 3434.08 and $2922.74 \mathrm{~cm}^{-1}$, respectively. Gum Ghatti's FTIR spectrum revealed broad peaks at $3425.65 \mathrm{~cm}^{-1}$ due to carbohydrate $\mathrm{O}-\mathrm{H}$ stretching, $2925.83 \mathrm{~cm}^{-1}$ due to $\mathrm{CH} 2$ asymmetric stretching, $1426.76 \mathrm{~cm}^{-1}$ due to $-\mathrm{CH} 2$ in-plane bending, $1070.65 \mathrm{~cm}^{-1}$ due to $-\mathrm{C}-\mathrm{O}$ stretching region as complex bands resulting from $\mathrm{C}-\mathrm{O}$ and $\mathrm{C}-\mathrm{O}-\mathrm{C}$ stretching vibrations, and $610.16 \mathrm{~cm}^{-1}$ due to pyranose ring (Fig. 2b). Gum Karaya's FTIR spectra revealed an absorption band at $3459.42 \mathrm{~cm}^{-1}$ relating to carbohydrate $\mathrm{O}-\mathrm{H}$ stretching, a peak at $2929.09 \mathrm{~cm}^{-1}$ due to $-\mathrm{CH} 2$ asymmetric stretching, and a strong band at $1428.15 \mathrm{~cm}^{-1}$ due to $-\mathrm{CH} 2$ in-plane bending (Fig. 2c). Due to $\mathrm{OH}$-outof-plane bending and NH-out-of-plane bending, it also showed maxima at $896.29 \mathrm{~cm}^{-1}$ and $780 \mathrm{~cm}^{-1}$, respectively. The absorption band at $3434.20 \mathrm{~cm}^{-1}$ related to $\mathrm{O}-\mathrm{H}$ stretching was demonstrated in the FTIR spectrum of dried blank Chitosan-Gellan Gum-Gum Ghatti beads (Fig. 2d). At $1730.14 \mathrm{~cm}^{-1}$, a novel band was discovered, which was linked to the presence of -COO- groups in blank Chitosan-Gellan Gum-Gum Ghatti beads. Furthermore, bands at $1450.67 \mathrm{~cm}^{-1}$ and $1620.09 \mathrm{~cm}^{-1}$ in Gellan Gum (spectra not shown) could be caused to asymmetric and symmetric stretching of - $\mathrm{COO}-$ groups, which were found moved to lower wave-numbers, showing participation of -COO- groups in the coordination process. The absorption band at $3415.14 \mathrm{~cm}^{-1}$ in the FTIR spectra of dried blank Chitosan-Gellan Gum-Gum Karaya beads corresponded to $\mathrm{O}-\mathrm{H}$ stretching (Fig. 2e).

Similarly, in the FTIR spectra of dried blank Chitosan-Gellan Gum-Gum Karaya beads, a novel band at $1739.77 \mathrm{~cm}^{-1}$ was observed, which might be attributable to the presence of -COO- groups. Bands at $1447.59 \mathrm{~cm}^{-1}$ and $1620.35 \mathrm{~cm}^{-1}$ in Gellan Gum (spectra not shown) could be due to asymmetric and symmetric stretching of COO-groups, which were shifted to lower wave-numbers, showing that -COO- groups were involved in the coordination process.

\section{Preparation of Chitosan-Gellan Gum-Gum Ghatti and -Gum Karaya PEC beads containing DS}

Extrusion of homogeneous dispersions of anionic polymerblends (Gellan Gum-Gum Ghatti or Gellan Gum-Gum Karaya) with DS into a gelation solution including chitosan (0.4 percent $\mathrm{w} / \mathrm{v}$, in 1 percent $\mathrm{w} / \mathrm{v}$ acetic acid) resulted in the formation of spherical PEC beads. Table 1 shows the formula for these DS-containing Chitosan-Gellan GumGum Ghatti and -Gum Karaya PEC beads. Figure 3 depicts photos of all of the Chitosan-Gellan Gum-Gum Ghatti/Gum Karaya PEC beads containing DS.

\section{Drug-excipient interaction analyses of Chitosan-Gellan Gum-Gum Ghatti and -Gum Karaya PEC beads containing DS}

DSC analyses were used to assess Chitosan-Gellan Gum-Gum Ghatti and -Gum Karaya PEC beads containing DS to see if there was any drug-excipient interaction. Figure 4 shows the DSC thermograms of DS, ChitosanGellan Gum-Gum Ghatti PEC beads containing DS (P4), and Chitosan-Gellan Gum-Gum Karaya PEC beads containing Diclofenac sodium (P6). The melting endotherms of pure DS were seen on a DSC thermogram at $294.23{ }^{\circ} \mathrm{C}$, which corresponded to its melting point (Fig. 4a). The endothermic peak was found at $227.20^{\circ} \mathrm{C}$ in the DSC thermogram of Chitosan-Gellan Gum-Gum Ghatti PEC beads containing DS (P4), which could be due to PEC matrix breakdown (Fig. 4b). Because of the poor heat transport across the stiff PEC network, the melting peak of the DS was pushed to a higher temperature $\left(317.06^{\circ} \mathrm{C}\right)$. The DSC thermogram of Chitosan-Gellan Gum-Gum Karaya PEC beads containing DS (P6), on the other hand, did not display DS melting endotherm, indicating that the medication was uniformly dispersed in the PEC network of the beads. However, it showed the endothermic peak at $225.63{ }^{\circ} \mathrm{C}$ corresponding to the degradation of PEC matrix.

\section{Drug entrapment efficiency}

Chitosan-based PEC beads containing DS had drug entrapment efficiency (\%) ranging from 59.54 2.09 to 81.034 .22 percent (Table 1). In Chitosan-Gellan GumGum Ghatti PEC beads containing DS, the greatest drug entrapment efficiency was recorded when the Gellan Gum to Gum Ghatti ratio was 1:1.5. (P3). When Gellan Gum to Gum Ghatti ratios of 1:1 (P2), 1:2 (P4), and 1.5:2.5 (P1) were used, the drug entrapment efficiencies were nearly comparable. The drug entrapment efficacy of Chitosan-Gellan Gum-Gum Karaya PEC beads containing DS enhanced when the concentration of Gum Karaya in the beads formula was increased (increased Gellan Gum to Gum Karaya ratio) (P5 and P6).

\section{In vitro swelling}

Table 1 shows the in vitro swelling patterns of several Chitosan-based PEC beads containing DS after $2 \mathrm{~h}$ in $0.1 \mathrm{M} \mathrm{HCl}(\mathrm{pH} 1.2)$ and $6 \mathrm{~h}$ in phosphate buffer $(\mathrm{pH} 6.8)$.

\section{In vitro drug release}

In vitro release of DS from different Chitosan-based PEC beads carrying DS was tested in phosphate buffer $(\mathrm{pH}$ 6.8) and revealed a consistent drug release profile over $6 \mathrm{~h}$. Figure 5 depicts the in vitro drug release pattern. 


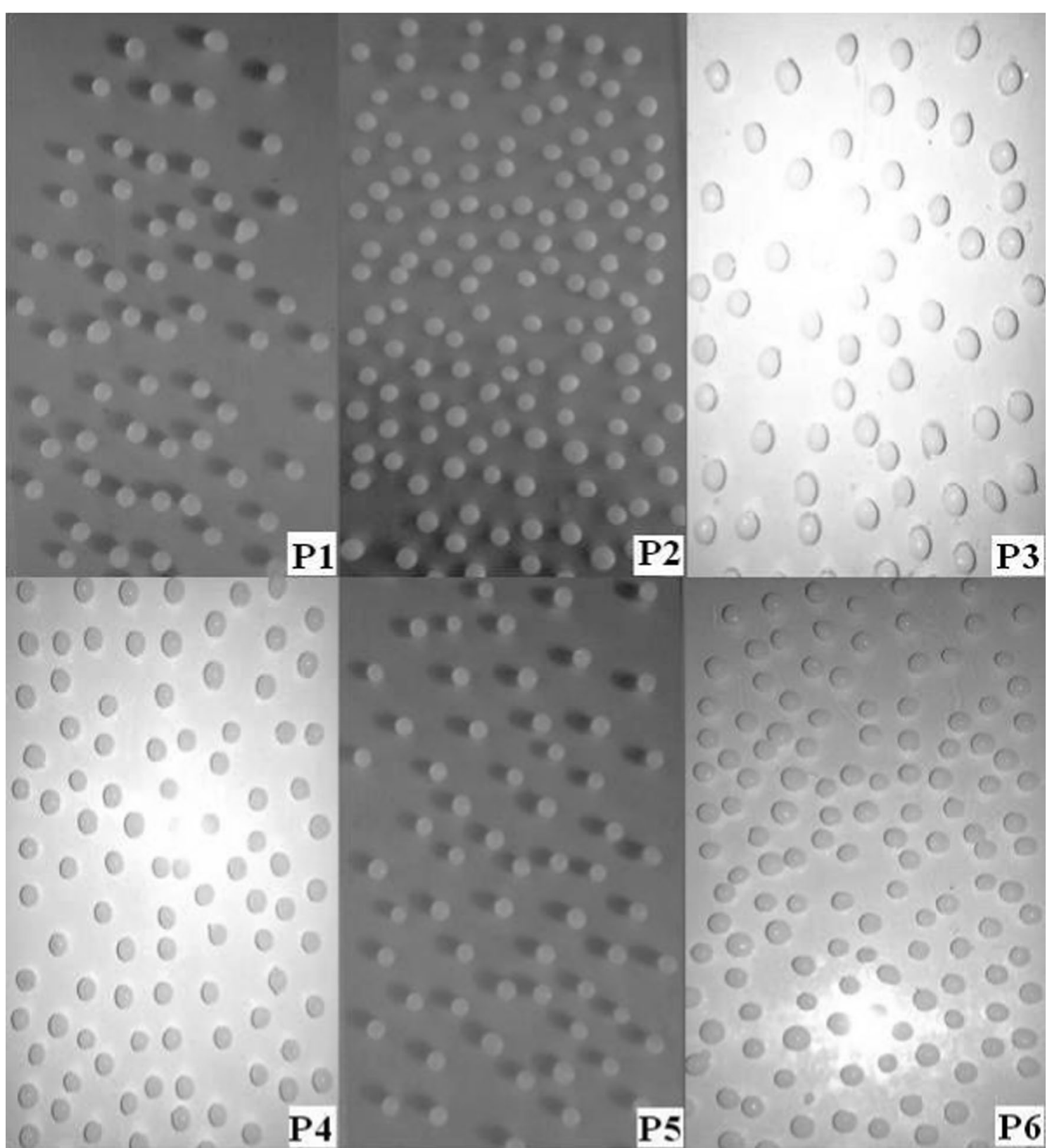

Fig. 3 Photographs of all these Chitosan-Gellan Gum-Gum Ghatti and -Gum Karaya PEC beads containing diclofenac sodium (P1 to P6)

The release data was subjected to the best-fitting evaluation using zero-order, first-order, Higuchi, and Korsmeyer-Peppas models to evaluate the release mechanism and kinetics of drug release from Chitosanbased PEC beads carrying DS (Tables 2 and 3). PEC beads formulations P1 and P2 followed the Higuchi release pattern, as indicated by their $\mathrm{r} 2$ values, based on kinetic analysis data (Table 2). According to the Higuchi model, cumulative drug release from a swelling hydrogel is proportional to the square root of time and is diffusion regulated. The conditions of pseudosteady state, constant diffusivity, rapid drug dissolution, and sinks are all assumed. The Higuchi model can be utilized if the release is primarily regulated by diffusion through water-filled pores in the matrix $[9,10]$. 

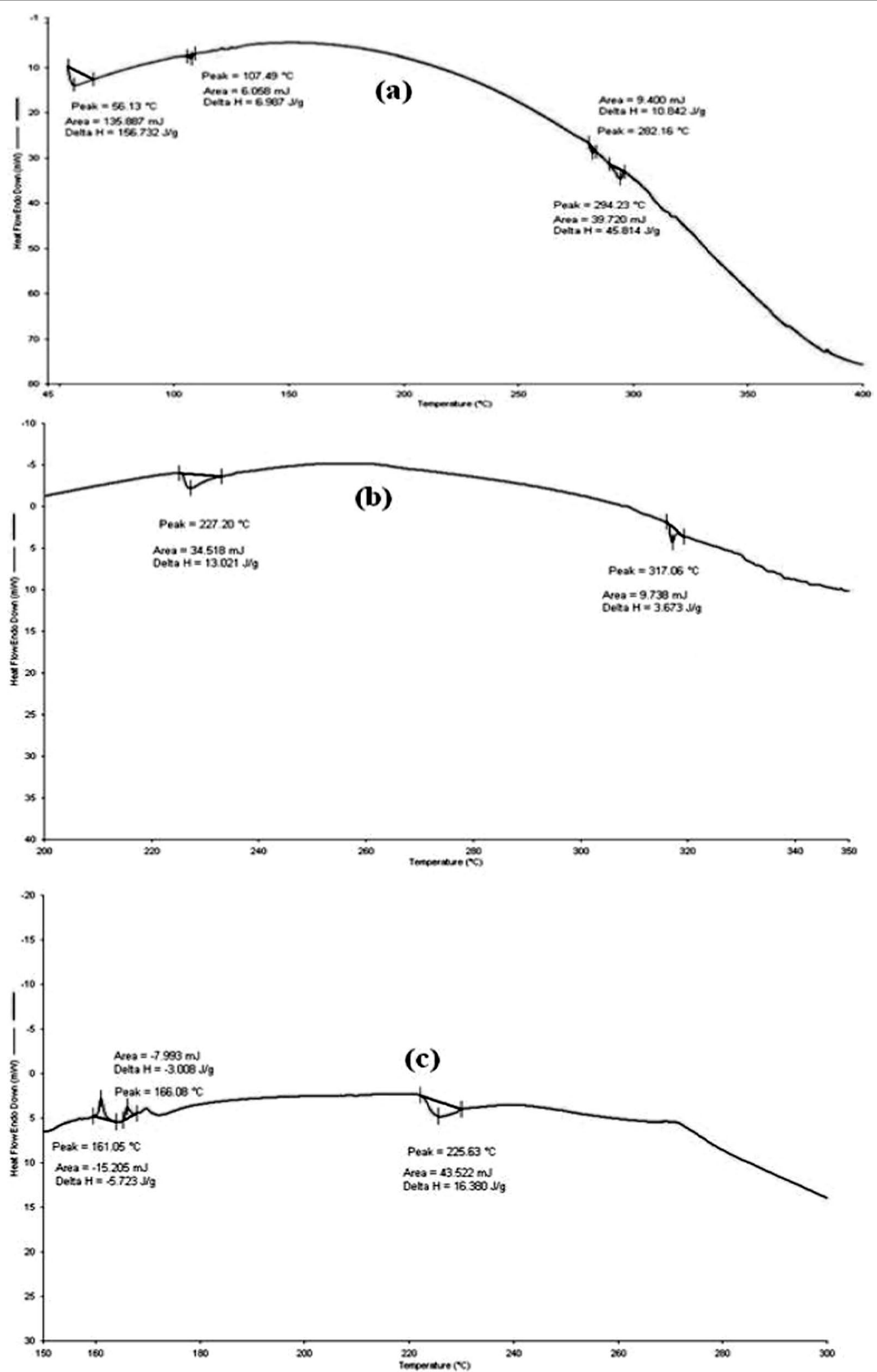

Fig. 4 DSC thermograms of pure Diclofenac sodium (a), P4 chitosan-Gellan gum-Gum Ghatti PEC beads containing Diclofenac sodium (b) and P6 Chitosan-Gellan Gum-Gum Karaya PEC beads containing Diclofenac sodium (c) 


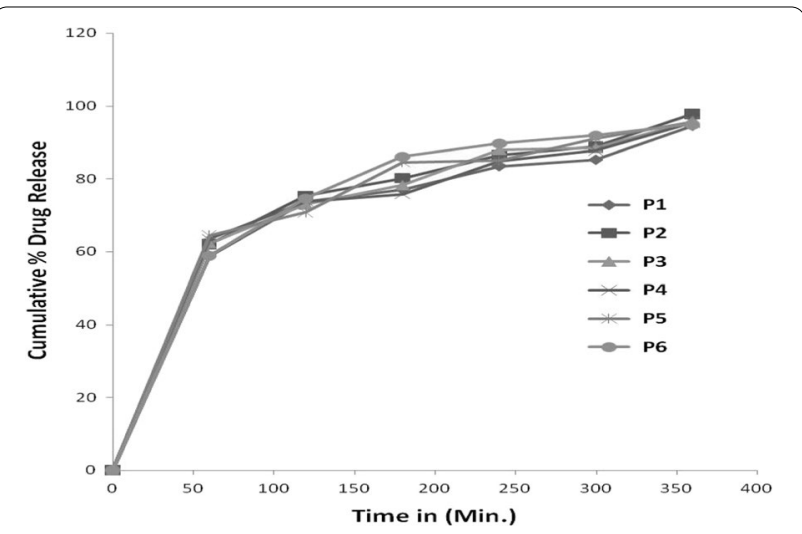

Fig. 5 In vitro release of Diclofenac sodium from various chitosan-based PEC beads containing Diclofenac sodium in phosphate buffer ( $\mathrm{pH} 6.8$ )

Table 2 Results of release kinetic models of various chitosanbased PEC beads containing Diclofenac sodium ( $1-6 \mathrm{~h}$ )

\begin{tabular}{|c|c|c|c|}
\hline \multirow{2}{*}{$\begin{array}{l}\text { Formulation } \\
\text { code }\end{array}$} & \multicolumn{3}{|l|}{$r^{2}$ value } \\
\hline & $\begin{array}{l}\text { Zero-order } \\
\text { model }\end{array}$ & First-order model & Higuchi model \\
\hline P1 & 0.9316 & 0.8971 & 0.9502 \\
\hline P2 & 0.9548 & 0.8398 & 0.9563 \\
\hline P3 & 0.9441 & 0.9274 & 0.9382 \\
\hline P4 & 0.9753 & 0.9039 & 0.9118 \\
\hline P5 & 0.9392 & 0.9533 & 0.9101 \\
\hline P6 & 0.8568 & 0.9931 & 0.9632 \\
\hline
\end{tabular}

Table 3 Results of Korsmeyer-Peppas models of various chitosan-based PEC beads containing Diclofenac sodium (10$60 \mathrm{~min})$

\begin{tabular}{lll}
\hline Formulation code & $\begin{array}{l}\boldsymbol{r}^{2} \text { value } \\
\text { Korsmeyer-Peppas model* }\end{array}$ & $\boldsymbol{n}$ value $^{*}$ \\
\hline P1 & 0.9802 & 0.97 \\
P2 & 0.9828 & 0.99 \\
P3 & 0.9904 & 0.98 \\
P4 & 0.9837 & 0.99 \\
P5 & 0.9823 & 0.99 \\
P6 & 0.9949 & 0.99 \\
\hline
\end{tabular}

*For drug released within first hour

\section{Discussion}

Chitosan (deacetylated chitin) is a cationic natural polysaccharide and is regarded as generally recognized as safe (GRAS) material [13]. It is composed of $\alpha$-1, 4-linked 2 - amino- 2-deoxy- $\alpha$-D-glucose ( $N$-acetyl glucosamine) [14]. Chitosan possesses excellent biocompatibility and biodegradability $[15,16]$. In drug delivery industry, chitosan has been exploited as a very promising biopolymer for use as carrier material for encapsulation of drugs [17, 18]. Though Chitosan is a promising carrier material in the formulation of polymer-based oral drug delivery systems, it displays poor mechanical behavior and limited capability for controlling drug release due to its greater solubility in lower $\mathrm{pH}$ through promoting faster dissolution of in the stomach $\mathrm{pH}$ [19]. The $\mathrm{pH}$-sensitivity of Chitosan is because of the protonation of free amino groups in response to external $\mathrm{pH}$ alterations [20]. Because Chitosan is a cationic polymer, it interacts with the negatively charged silicic acid residues in mucin, the glycoprotein that makes up mucus [21]. Chitosan's mucoadhesive properties are due to this interaction. Additionally, Chitosan's hydroxyl and amino groups may form hydrogen bonds with mucus [22]. During past few years, numerous Chitosan-based PECs with a number of anionic polymers are being researched as drug delivery carrier materials by several research groups $[1,2,6,23]$. In the present investigation, PEC beads were prepared using cationic Chitosan and anionic polymer-blends of Gellan GumGum Ghatti or Gellan Gum-Gum Karaya for prolonged drug release. DS, a non-steroidal anti-inflammatory drug (NSAID) of about 1-2 h biological half-life [9, 12, 24], was investigated as model drug to assess the prolonged drug release properties. The proposed Chitosan-based PEC beads were expected to retard the release of encapsulated DS in alkaline $\mathrm{pH}$ (6.8) with minimal release in the acidic environment of stomach ( $\mathrm{pH} 1.2)$.

\section{Confirmation of Chitosan-Gellan Gum-Gum Ghatti and-Gum Karaya PEC beads formation}

Because of the protonation of amino groups on the backbone, chitosan becomes a cationic polyelectrolyte in acidic medium, which is able to form PECs with negatively charged polyelectrolytes [23]. Considering the versatility of Chitosan-based PECs, in the present study, an attempt was made to prepare PEC beads comprised of cationic Chitosan and anionic polymers like Gum Karaya and Gum Ghatti in combination with Gellan Gum. During the preliminary trial experimentation, it was observed that PEC beads was formed using Chitosan along with Gum Ghatti-Gum Karaya blends were very weak; therefore, to provide mechanical strength to the beads, Gellan Gum was added into the system. For the confirmation of PEC formation, these formed spherical beads were characterized by DSC analyses and FTIR Spectroscopy analyses. 


\section{DSC analysis}

\section{Chitosan-Gellan Gum-Gum Ghatti beads}

The change(s) in appearance and temperature of the endothermic peak(s) in the DSC thermograms of dried blank Chitosan-Gellan Gum-Gum Ghatti beads compared to DSC thermograms of Chitosan, Gellan Gum and Gum Ghatti suggested the formation of new polymeric system of PEC between Cationic Chitosan and anionic Gellan Gum-Gum Ghatti.

\section{Chitosan-Gellan Gum-Gum Karaya beads}

Similarly, when comparing DSC thermograms of dried Chitosan-Gellan Gum-Gum Karaya beads to DSC thermograms of Chitosan, Gellan Gum, and Gum Karaya, the change(s) in appearance and temperature of the endothermic peak(s) in the DSC thermograms of dried Chitosan-Gellan Gum-Gum Karaya beads suggested the formation of PEC between cationic Chitosan and anionic Gellan Gum-Gum Karaya.

\section{Fourier transform-infrared (FTIR) spectroscopy analyses Chitosan-Gellan Gum-Gum Ghatti beads}

The FTIR spectrum of dried blank Chitosan-Gellan Gum-Gum Ghatti beads showed shifting of bands due to amide I of Chitosan to lower wave-number, whereas bands due to amide II and III were found disappeared in the FTIR spectrum of dried blank Chitosan-Gellan Gum-Gum Ghatti beads. This phenomena suggested PEC formation between cationic Chitosan and anionic polymers (here Gellan Gum-Gum Ghatti) in the dried blank Chitosan-Gellan Gum-Gum Ghatti beads.

\section{FTIR spectrum of dried blank Chitosan-Gellan Gum-Gum Karaya beads}

The band due to amide I of Chitosan was found to be shifted to a lower wave-number in the FTIR spectrum of dried blank Chitosan-Gellan Gum-Gum Karaya beads, whereas bands due to amide II and III were found to have vanished. These shifting and disappearances of bands suggested PEC formation between cationic Chitosan and anionic polymers (here Gellan Gum-Gum Karaya) in the dried blank beads.

Preparation of Chitosan-Gellan Gum-Gum Ghatti and-Gum Karaya PEC beads containing DS

During the preparation of beads, it was observed that when DS was added to the homogenous aqueous dispersion of anionic polymer(s), the viscosity of the system decreased and the system became fluid. This has resulted in easy extrusion of drug-polymer liquid mix through the hypodermic syringe even though high concentrations of anionic polymer were used. This could be explained as when Gellan gum/Gum Ghatti or Gellan gum /Gum
Karaya were added to the deionized water, the polymer blend became fully hydrated due to application of heat $\left(90{ }^{\circ} \mathrm{C}\right)$. This has resulted in their ionization $(\mathrm{pH}$ of aqueous dispersion was around 6.0) and because of this they carry a negative charge due to $\mathrm{COO}^{-}$. This led to increased charge density and thus increased electrostatic repulsion leading to thicker hydrogel.

Diclofenac, 2-\{2-[(2, 6-dichloro phenyl) amino] phenyl $\}$ acetic acid, is a weak acid having the pKa of 4.15 [24]. It is more soluble in alkaline $\mathrm{pH}$ than acidic $\mathrm{pH}$. Its solubility at neutral $\mathrm{pH}$ is around $1.13 \mathrm{~g} / \mathrm{L}$ (DS) [25]. In the present study, the $\mathrm{pH}$ of the aqueous dispersion of polymeric blends was 6.0, which is $2 \mathrm{pH}$ units above the pKa of Diclofenac. Therefore, more of the drug is expected to be ionized as Diclofenac anions. This might have resulted in a decrease in degree of swelling due to the screening of electrostatic repulsion between charged groups on the polymer chains and, thus, a reduction in viscosity.

\section{Drug-excipient interaction analyses of Chitosan-Gellan Gum-Gum Ghatti and -Gum Karaya PEC beads containing DS}

No drug-polymer interaction was observed in both the chitosan-based PEC beads containing DS.

\section{Drug entrapment efficiency}

Highest drug entrapment efficiency (\%) was found in case of P6 Chitosan-Gellan Gum-Gum Karaya PEC beads containing DS $(81.03 \pm 4.22 \%)$. This occurrence could be attributed to the increased viscosity of the drug-polymer mixture, which hindered drug migration towards the acidic Chitosan-based gelation medium during the formation of Chitosan-Gellan Gum-Gum Karaya PEC beads containing DS. The high drug entrapment efficiencies shown by the Chitosan-based PEC beads P3 and P6 could also be attributed to the stoichiometry related with the polyanion to polycation ratio, which might provide strong PEC structure that could entrap more amount of the drug.

\section{In vitro swelling}

Overall swelling index values of these Chitosan-based PEC beads were found low. This could be attributed to the formation of more compact structure due to PEC formation. The Chitosan-based PEC beads swelled to a significantly greater extent at $\mathrm{pH}$ 6.8. The fluctuation in the degree of ionization of functional groups controlled by the $\mathrm{pH}$ of the swelling media could explain the $\mathrm{pH}$ responsive swelling behavior of these PEC beads. An acid-base type PEC system is formed when the negatively charged -COO- groups of anionic polysaccharide(s) bond to the positively charged amino groups of Chitosan in an acidic solution. The amino groups are deionized 
when the $\mathrm{pH}$ rises, and the binding affinity between two polyelectrolyte molecules decreases, causing the PEC to inflate and disintegrate, modulating the release. [26]. This $\mathrm{pH}$ responsive swelling behavior should be advantageous for the PEC beads in sustained drug release applications, where inhibition of the drug release in the gastric environment (low $\mathrm{pH}$ values) is desired. It is possible to precisely tune medication release at the target spot by altering elements that produce PEC's swelling features [26].

\section{In vitro drug release}

The in vitro release of DS from various PEC bead formulations was found faster initially. Within the first hour, about $59-64 \%$ of the entrapped drug were found to be released from these Chitosan-based PEC beads containing DS. The ionic interactions between cationic Chitosan and anionic polymer-blends might have been reduced at $\mathrm{pH} 6.8$ forming a loose network of the tested beads with increased porous surface, which could allow a large amount of dissolution medium to enter into the PEC matrix. This might have resulted in rapid dissociation of the PEC bead-matrix, leading to drug release with a burst effect. Another possible explanation of the initial burst drug release from these PEC beads within the first hour of drug release study could most likely be due to the presence of drug crystals onto bead surface. The drug crystals might be formed onto these PEC bead surfaces because of their migration along with water to the surface during drying [27]. At pH 6.8, because of higher solubility of entrapped drug (here DS) and suppressed ionization of polyelectrolytes within these formulated PEC beads, the in vitro drug release was found increased $[1,18]$. After the first hour, comparatively slower DS release was observed. About 94 to $97 \%$ of in vitro DS release was released from these Chitosan-based PEC beads at the end of $6 \mathrm{~h}$. The release kinetics of the PEC bead formulations P3-P5 were zero-order. When a release rate is basically constant over time, the zero-order release is appropriate. The PEC bead formulation P6 followed first-order drug release pattern. In this case, an initial burst release effect was evidenced where the entrapped drug (here DS) was being rapidly dissolved and released from the bead-surface before the formation of gel-layer. After that the drug release rate of PEC bead formulation P6 was found decreased continually until the end of the drug release process. Since more than $60 \%$ of DS were released within the first hour, then values were calculated by Korsmeyer-Peppas model using drug release result up to first hour (Table 3). The $n$ value of different PEC bead formulations ranged between 0.97 and 0.99 , indicating a super case-II transport mechanism. This type of release describes a transport in which the rate of dissolution medium uptake into the polymer matrix is substantially dictated by the rate of swelling and relaxation of the polymer chains, and in which the drug diffusion varies on both concentration and time.

\section{Conclusions}

In the present investigation, an attempt has been made to formulate and evaluate PEC beads containing DS, which were made of cationic Chitosan and anionic polymer-blends of Gellan Gum-Gum Ghatti or Gellan Gum-Gum Karaya. When the mixtures of homogenous dispersions of Gellan Gum-Gum Ghatti or Gellan Gum-Gum Karaya with DS were extruded into the gelation medium composed of Chitosan $(0.4 \% \mathrm{w} / \mathrm{v}$, in $1 \% \mathrm{w} / \mathrm{v}$ acetic acid), spherical PEC beads containing DS were formed instantaneously due to electrostatic interaction between oppositely charged polymers. The PEC formation between cationic and anionic polymers in these newly formulated Chitosan-based beads were suggested by DSC and FTIR analyses. No drug-polymer interaction was observed in these Chitosan-based PEC beads containing DS and this was suggested by the results of DSC analyses. The drug entrapment efficiencies (\%) of these PEC beads containing DS ranged from $59.54 \pm 2.09$ to $81.03 \pm 4.22 \%$. Highest drug entrapment efficiency (\%) was found in case of P6 ChitosanGellan Gum-Gum Karaya PEC beads containing DS $(81.03 \pm 4.22 \%)$. All these Chitosan-based PEC beads containing DS exhibited $\mathrm{pH}$ responsive swelling behavior when tested in vitro. The in vitro release of DS from various Chitosan-based PEC beads in phosphate buffer (pH 6.8) showed a sustained drug releasing profile over $6 \mathrm{~h}$. From the experimental data, it is clear that these types of Chitosan-Gellan Gum-Gum Ghatti and -Gum Karaya PEC beads can be useful as potential multipleunit polymeric carrier systems for sustained delivery of gastric irritant drugs like Diclofenac sodium.

\section{Abbreviations \\ GIT: Gastrointestinal tract; PEC: Polyelectrolyte complex; CH: Chitosan; GG: Gellan Gum; GT: Gum Ghatti; GK: Gum Karaya; DS: Diclofenac Sodium; NSAIDs: Nonsteroidal anti-inflammatory drugs; OA: Osteoarthritis; RA: Rheumatoid arthritis.}

\section{Acknowledgements}

The authors express their sincere thanks to Prof. Raghubir Singh, Vice Chancellor, Teerthanker Mahaveer University, Moradabad, Uttar Pradesh, India, for his constant encouragement to carry out this work.

\footnotetext{
Authors' contributions

PK and VR conceived and designed the study. PM, VR and PK involved in the preparation of polymer solutions, experimentations and contributed to the preparation of the manuscript. AV performed experimental analysis of the data and contributed to the preparation of the manuscript. PM critically reviewed the entire manuscript. AV supervised the study, involved in the design and execution of the study. All authors discussed in the manuscript and approved its final version. All authors have been personally and actively
} 
involved in substantial work leading to the paper, and will take public responsibility for its content.

\section{Funding}

The author(s) received no specific funding for this work.

\section{Availability of data and materials}

All data and material are available upon request.

\section{Declarations}

Ethics approval and consent to participate

Not applicable.

\section{Consent of publication}

Not Applicable.

\section{Competing interests}

The authors declare that they have no competing interests.

\section{Author details}

${ }^{1}$ Teerthanker Mahaveer College of Pharmacy, Teerthanker Mahaveer University, Moradabad, Uttar Pradesh 244001, India. ${ }^{2}$ Department of Pharmaceutics, School of Pharmaceutical Sciences, IFTM University, Moradabad 244001, India.

Received: 24 June 2021 Accepted: 22 September 2021

Published online: 02 October 2021

\section{References}

1. Bickley LK, van Aerle R, Brown AR, Hargreaves A, Huby R, Cammack V, Jackson R, Santos EM, Tyler CR (2017) Bioavailability and kidney responses to diclofenac in the fathead minnow (Pimephales promelas). Environ Sci Technol 51(3):1764-1774

2. Scholer DW, Ku EC, Boettcher I, Schweizer A (1986) Pharmacology of diclofenac sodium. Am J Med 80(4B):34-38

3. Willis JV, Kendall MJ, Flinn RM, Thornhill DP, Welling PG (1979) The pharmacokinetics of diclofenac sodium following intravenous and oral administration. Eur J Clin Pharmacol 16(6):405-410

4. Kendall MJ, Thornhill DP, Willis JV (1979) Factors affecting the pharmacokinetics of diclofenac sodium (Voltarol). Rheumatol Rehabil 2:38-46

5. Laine L, Connors LG, Reicin A, Hawkey CJ, Burgos-Vargas R, Schnitzer TJ, Yu Q, Bombardier C (2003) Serious lower gastrointestinal clinical events with nonselective NSAID or coxib use. Gastroenterology 124(2):288-292

6. Kumar A, Ahuja M (2013) Carboxymethyl gum kondagogu-chitosan polyelectrolyte complex nanoparticles: preparation and characterization. Int J Biol Macromol 62:80-84

7. Luo Y, Wang Q (2014) Recent development of chitosan-based polyelectrolyte complexes with natural polysaccharides for drug delivery. Int J Biol Macromol 64:353-367

8. Dixit R, Verma A, Soni S, Mishra A, Bansal A, Pandit J (2011) Preparation and characterization of gellan-chitosan polyelectrolyte complex beads. Lat Am J Pharm 30:1186-1195

9. Verma A, Nagarwal RC, Sharma SD, Pandit JK (2012) Preparation and characterization of floating gellan-chitosan polyelectrolyte complex beads. Lat Am J Pharm 31:138-146

10. Thiinemann AF, Müller M, Dautzenberg H, Joanny JF, Löwen H (2004) Polyelectrolyte complexes. In: Polyelectrolytes with defined molecular architecture II. Springer, Berlin

11. Malakar J, Nayak AK, Jana P, Pal D (2013) Potato starch-blended alginate beads for prolonged release of tolbutamide: development by statistical optimization and in vitro characterization. Asian J Pharm 7:43-51
12. Nayak AK, Pal D, Santra K (2016) Swelling and drug release behavior of metformin $\mathrm{HCl}$-loaded tamarind seed polysaccharide-alginate beads. Int J Biol Macromol 82:1023-1027

13. Nayak A, Pal D, Malakar J (2013) Development, optimization, and evaluation of emulsion-gelled floating beads using natural polysaccharideblend for controlled drug release. Polym Eng Sci 53:238-250

14. Hasnain MS, Nayak AK, Singh M, Tabish M, Ansari MT, Ara TJ (2016) Alginate-based bipolymeric-nanobioceramic composite matrices for sustained drug release. Int J Biol Macromol 83:71-77

15. Guru PR, Nayak AK, Sahu RK (2013) Oil-entrapped sterculia gum-alginate buoyant systems of aceclofenac: development and in vitro evaluation. Colloids Surf B Biointerfaces 104:268-275

16. Sinha P, Ubaidulla U, Hasnain MS, Nayak AK, Rama B (2015) Alginate-okra gum blend beads of diclofenac sodium from aqueous template using $\mathrm{ZnSO}_{4}$ as a cross-linker. Int J Biol Macromol 79:555-563

17. Jana S, Maji N, Nayak A, Sen K, Basu SK (2013) Development of chitosanbased nanoparticles through inter-polymeric complexation for oral drug delivery. Carbohydr polym 98(1):870-876

18. Jana S, Manna S, Nayak AK, Sen KK, Basu SK (2014) Carbopol gel containing chitosan-egg albumin nanoparticles for transdermal aceclofenac delivery. Colloids Surf B Biointerfaces 114:36-44

19. Hasnain MS, Nayak AK, Singh R, Ahmad F (2010) Emerging trends of natural-based polymeric systems for drug delivery in tissue engineering applications. Sci J UBU 1(2):1-13

20. Nayak AK, Pal D (2012) Natural polysaccharides for drug delivery in tissue engineering. Everyman's Sci 46:347-352

21. Prabaharan M, Mano JF (2005) Chitosan-based particles as controlled drug delivery systems. Drug Deliv 12(1):41-57

22. Bhise KS, Dhumal RS, Chauhan B, Paradkar A, Kadam SS (2007) Effect of oppositely charged polymer and dissolution medium on swelling, erosion, and drug release from chitosan matrices. AAPS PharmSciTech 8(2): Article 44

23. Nayak AK, Pal D (2015) Chitosan-based interpenetrating polymeric network systems for sustained drug release. In: Advanced theranostic materials. Scrivener Publishing LLC, USA

24. Nayak AK (2010) Thermodynamic study of the diclofenac sodium solubility in various oils. Khimiya 19:121-128

25. Martindale W, Reynolds JEF, Royal Pharmaceutical Society of Great Britain (1996) Martindale: the extra pharmacopoeia. Royal Pharmaceutical Society, London

26. Sonia TA, Sharma CP (2011) Chitosan and its derivatives for drug delivery perspective. In: Chitosan for biomaterials I. Springer, Berlin

27. Calija B, Cekic N, Milic J (2015) Chitosan-based polyelectrolyte complexes: characteristics and application in formulation of particulate drug carriers. In: Advanced theranostic materials. Scrivener Publishing LLC, USA

\section{Publisher's Note}

Springer Nature remains neutral with regard to jurisdictional claims in published maps and institutional affiliations.

\section{Submit your manuscript to a SpringerOpen ${ }^{\circ}$ journal and benefit from:}

- Convenient online submission

- Rigorous peer review

- Open access: articles freely available online

- High visibility within the field

- Retaining the copyright to your article

Submit your next manuscript at $\boldsymbol{\nabla}$ springeropen.com 